\title{
Perfil de morbidade de crianças hospitalizadas em um hospital público: implicações para a Enfermagem
}

\author{
Profile of morbidity of children hospitalized in a public hospital: implications for Nursing \\ Perfil de morbilidad de niños hospitalizados en un hospital público: consecuencias para la Enfermería
}

\section{Beatriz Rosana Gonçalves de Oliveira', Cláudia Silveira Viera", Maria Cândida de Carvalho Furtado"', Débora Faleiros de Mello"', Regina Aparecida Garcia de Lima"'I}

\author{
' Universidade Estadual do Oeste do Paraná, Curso de Enfermagem. Cascavel-PR, Brasil. Universidade de São Paulo, \\ Escola de Enfermagem de Ribeirão Preto, Programa de Pós Graduação em Enfermagem em Saúde Pública (Doutoranda). \\ Ribeirão Preto-SP, Brasil. \\ "Universidade Estadual do Oeste do Paraná, Curso de Enfermagem. Cascavel-PR, Brasil. \\ "' Universidade de São Paulo, Escola de Enfermagem de Ribeirão Preto. Ribeirão Preto-SP, Brasil.
}

Submissão: 25-02-2011 Aprovação: 12-09-2012

\section{RESUMO}

Objetivou-se conhecer as causas de hospitalização de crianças menores de cinco anos num hospital público no Paraná, para subsidiar as ações de enfermagem. Realizou-se uma pesquisa quantitativa, descritiva, exploratória, com análise estatística inferencial. Foram analisados 722 prontuários, no período de 2005-2009. Observou-se que menores de um ano tiveram mais hospitalizações $(42 \%)$ do que os de um a cinco anos; maioria de internações do sexo masculino (65\%); doenças respiratórias predominaram (56\%) em relação ao conjunto das demais; tempo médio de internação de 9,4 dias para menores de um ano e 8,3 dias para menores de cinco anos; 95\% tiveram alta hospitalar como desfecho. Esse perfil auxilia a direcionar o cuidado de enfermagem, incorporando os dados no planejamento, tanto na atenção básica quanto no hospital.

Descritores: Hospitalização; Morbidade; Serviços de Saúde; Criança.

\begin{abstract}
One aimed to know the causes of hospitalization among children under five years in a public hospital in Paraná to subsidize nursing actions. A descriptive, quantitative, exploratory study was carried out, with inferential statistical analysis. One assessed 722 medical records from 2005-2009. It was observed that children under one year had more hospitalizations (42\%) than those with one to five years; a higher percentage of male admissions (65\%); respiratory diseases predominated (56\%) compared to all other; mean length of stay of 9.4 days for those under one year and 8.3 days for children under five years; $95 \%$ were discharged as the outcome. This profile helps to direct nursing care, incorporating the data in planning, both in primary care and in hospital. Key words: Hospitalization; Morbidity; Health services; Child.
\end{abstract}

\section{RESUMEN}

Objetivó-se conocer las causas de hospitalización entre los niños menores de cinco años en un hospital público de Paraná para subvencionar las acciones de enfermería. Realizó-se una pesquisa cuantitativa, descriptiva, exploratoria, con análisis estadística inferencial. Se evaluaron 722 historias clínicas, no período de 2005-2009. Observó-se que: menores de un año habían sido más hospitalizados (42\%) que aquellos con uno a cinco años; una mayor porcentaje de hospitalizaciones de niños del sexo masculino (65\%); las enfermedades respiratorias predominaron (56\%) en comparación con todas las otras; y una permanencia media hospitalaria de 9,4 días en menores de un año, y de 8,3 días para los niños menores de cinco años; 95\% fueron dados de alta en el resultado. Este perfil ayuda a organizar la atención directa de enfermería, incorporando-se los datos en la planificación, tanto en atención primaria cuanto en el hospital.

Palabras clave: Hospitalización; Morbilidad; Servicios de Salud; Niño. 


\section{INTRODUÇÃO}

Crianças são mais susceptíveis ao agravamento de uma enfermidade em função da fragilidade própria do extremo da idade. Para tanto, a atenção à saúde desse grupo deve ser compreendida como um campo prioritário para as ações da atenção primária em saúde, visto que pode contribuir para o desenvolvimento de estratégias de enfrentamento de problemas de morbidade, mortalidade e qualidade de vida da população infantil. Apesar dos esforços e avanços alcançados no setor saúde, alguns indicadores de saúde da criança, tal como a morbidade infantil, apontam para problemas que precisam de ações, práticas e intervenções que sejam resolutivas.

Estudar as causas de internação hospitalar em crianças menores de cinco anos pode nos auxiliar a compreender o perfil de adoecimento nessa faixa etária e elaborar planos de atenção à saúde que previnam o agravamento das doenças a fim de que a hospitalização seja evitada e, quando isso não for possível, direcionar as ações da equipe de enfermagem para o planejamento de um cuidado mais efetivo no âmbito do hospital, minimizando as consequências da hospitalização.

Para a elaboração desses planos de cuidado, a Enfermagem utiliza-se da Sistematização da Assistência de Enfermagem - SAE, que contribui para organizar o cuidado, tornando possível a operacionalização do Processo de Enfermagem. Tal proposta dá visibilidade as ações da enfermagem no âmbito da atenção à saúde, em qualquer ambiente onde a prática profissional ocorra, seja em instituições prestadoras de serviços de internação hospitalar ou em serviços ambulatoriais, assim como outros espaços de atuação da profissão ${ }^{(1)}$.

Para dar sustentação ao planejamento das ações, os dados epidemiológicos são instrumentos importantes, pois, auxiliam a fundamentar a ação de enfermagem e o aperfeiçoamento de competência para aplicação, de modo planejado e dinâmico, do processo assistencial, permitindo identificar, compreender, descrever, explicar e predizer quais as necessidades da pessoa, família ou coletividade humana, em determinado momento do processo saúde e doença ${ }^{(1)}$.

Muitos programas de atenção a saúde da criança vêm sendo propostos e alguns desenvolvidos em amplo espectro no Brasil, tanto na atenção básica quanto especializada, com a participação dos enfermeiros, a exemplo da estratégia "Brasileirinhos e brasileirinhas saudáveis: primeiros passos para o desenvolvimento nacional", aprovado pela portaria GM $2395 / 2009^{(2)}$, contudo, é preciso acompanhar o impacto dessas ações não somente avaliando os programas de atenção básica, mas também seu reflexo, por meio da avaliação da morbidade das crianças.

Editorial da revista Cadernos de Saúde Pública(3) registra que as infecções respiratórias agudas persistem como problema de saúde pública. Indica que o Brasil é um dos países com maior número de casos anuais de pneumonia clínica em menores de cinco anos, com incidência estimada de 0,11 episódios por criança/ano. Destas, 30 a 50\% das consultas ambulatoriais, mais de 50\% das hospitalizações e de 10 a 15 \% dos óbitos são atribuídos as infeções respiratórias agudas, sendo que $80 \%$ às pneumonias.
Apesar das reconhecidas limitações dos dados de morbidade para a caracterização dos problemas de saúde de uma população, devido ao seu caráter seletivo (acesso ao serviço, somente hospitalizações em hospital público, oferta de leitos hospitalares, para citar apenas alguns) sua análise tem sido útil em avaliações dos serviços de saúde no Brasil( ${ }^{(4)}$, assim como para a formulação e o aperfeiçoamento de políticas de saúde e estratégias de cuidado. Os registros dos serviços de saúde são importantes fontes de dados para a análise da morbidade, servindo como indicador de saúde da população usuária daquele serviço e ainda são úteis para o planejamento das ações de saúde a serem desenvolvidas nos diversos serviços e programas de saúde. Assim, torna-se importante conhecer o perfil de morbidade das crianças menores de cinco anos, com o objetivo de preparar os serviços para as novas necessidades ou expectativas.

Buscando contribuir com tal discussão, o presente estudo tem por objetivo conhecer as causas de hospitalização de crianças menores de cinco anos num hospital público no Paraná para subsidiar as ações de enfermagem, tanto no âmbito da atenção básica quanto hospitalar.

\section{MÉTODO}

Pesquisa quantitativa, descritiva, exploratória e inferencial, cujos dados foram colhidos junto ao Serviço de Arquivo Médico e Estatística - SAME do Hospital Universitário do Oeste do Paraná - HUOP, em prontuários das crianças menores de cinco anos, hospitalizadas no Alojamento Conjunto PediátriCo - ACP, no período de junho de 2005 a junho de 2009. O total de prontuários no período foi de 3942, referentes às internações de zero a quatorze anos. Nesse estudo, na faixa etária de interesse, as crianças menores de cinco anos, corresponderam a $2638(66,92 \%)$ hospitalizações, das quais, para a amostragem, considerou-se 95\% de confiança e 3\% de margem de erro, obtendo-se uma amostra de 722 prontuários.

Selecionou-se os prontuários mediante amostragem sistemática, no caso, a cada sete prontuários, um foi selecionado, para identificar as variáveis: diagnóstico, sexo, idade, procedência, tempo de permanência e tipo de saída. O período de coleta compreendeu duas semanas em horário integral, com oito horas de coleta por dia, com o auxílio de seis alunas do Curso de Graduação em Enfermagem da Universidade Estadual do Oeste do Paraná, treinadas e supervisionadas pela pesquisadora durante todo o período.

A classificação de faixa etária adotada para divisão do grupo em menor de um ano incluiu crianças de zero a onze meses e 29 dias, hospitalizadas na unidade de ACP da instituição do estudo. Aquelas crianças no período neonatal, hospitalizadas na unidade de terapia intensiva neonatal, oriundas da própria instituição ou transferidas de outros serviços não foram incluídas na amostra, bem como aquelas hospitalizadas na unidade de cuidados intermediários. A faixa etária de um a menores de cinco anos incluiu as crianças com um ano completo até quatro anos, onze meses e 29 dias, também, hospitalizadas na mesma unidade, excluindo-se as internações na unidade de terapia intensiva pediátrica. 
Ainda, optou-se por trabalhar com a informação obtida da guia de autorização para internação hospitalar - AlH para obtenção do diagnóstico inicial, padronizando o documento de obtenção dessa informação, na tentativa de torná-lo o mais fidedigno possível.

Transcreveram-se os dados em tabelas e analisou-se utilizando da estatística descritiva com o cálculo de porcentagem e média, da estatística inferencial paramétrica com teste t-student para a comparação de médias de duas amostras independentes e estatística inferencial não paramétrica com teste qui-quadrado, ambos considerando 5\% de significância.

Os dados apresentados nesse estudo são parte de um estudo maior intitulado "Resolutividade do atendimento a saúde das crianças menores de cinco anos com queixas de doenças evitáveis", aprovado pelo Comitê de Ética em Pesquisa da Universidade Estadual do Oeste do Paraná - UNIOESTE, sob parecer no 245/2009 - CEP.

\section{RESULTADOS}

Apresenta-se a distribuição em menores de um ano e de um a menores de cinco anos, segundo o sexo, na tabela 01. Para essa comparação utilizou-se o Teste qui-quadrado, sendo possível demonstrar que foi significativamente maior a porcentagem de internações do sexo masculino nas duas faixas etárias.

Tabela 1 - Distribuição de menores de cinco anos, segundo o sexo, nas hospitalizações do ACP do HUOP, no período de 2005 a 2009, Cascavel, PR, 2009.

\begin{tabular}{|c|c|c|c|c|}
\hline \multirow{2}{*}{ Faixa Etária } & \multicolumn{2}{|c|}{ Sexo } & \multirow[b]{2}{*}{ Total } & \multirow[b]{2}{*}{ p-valor } \\
\hline & Feminino & Masculino & & \\
\hline \multirow{3}{*}{$<1$ ano } & 166 & 234 & 400 & \multirow{3}{*}{$0,00 *$} \\
\hline & & & & \\
\hline & $42 \%$ & $59 \%$ & $100 \%$ & \\
\hline \multirow{3}{*}{$1 \mathrm{a}<5$ anos } & 117 & 205 & 322 & \multirow{3}{*}{$0,00^{*}$} \\
\hline & & & & \\
\hline & $36 \%$ & $64 \%$ & $100 \%$ & \\
\hline
\end{tabular}

*Significativo ao nível de $5 \%$ de probabilidade
Para avaliar a significância de um grupo que chamou a atenção por sua predominância na análise descritiva, efetuou-se o Teste qui-quadrado para comparação entre as porcentagens de hospitalizações por causas respiratórias com o conjunto de todas as outras causas, excluído o grupo das demais causas definidas, que compreendeu todos os diagnósticos isolados somados. Assim, na tabela 2 apresenta-se essa comparação.

Ao analisar o dado isoladamente, encontrou-se significância maior para as doenças respiratórias apenas no ano de 2008. Ao contrário, analisando o conjunto dos dados no período estudado, as doenças respiratórias são significativas como causa de internação em relação ao conjunto de todas as demais causas somadas.

Encontrou-se como primeiro motivo de internação hospitalar em crianças menores de cinco anos as doenças do aparelho respiratório, com média de 49,6\%. Estão incluídas nesse agrupamento as doenças do aparelho respiratório não crônicas, descritas no laudo médico para solicitação de Autorização de Internação Hospitalar- AlH como broncopneumonia (BCP), pneumonia (PMN), insuficiência respiratória aguda (IRA), infecção das vias aéreas (IVAS), bronquiolite, otite média aguda (OMA), entre outras menos frequentes.

Os demais 51,4\% são referentes a outras patologias, incluindo também aquelas consideradas como sensíveis ao cuidado na atenção básica, como as doenças gastrointestinais, desidratação e desnutrição, com um percentual médio de 6,5\%. As causas neonatais conduziram a morbidade hospitalar com média de 8,5\%, as causas externas (queimaduras, intoxicação exógena, acidentes automobilísticos, quedas de altura, atropelamentos), com $2,4 \%$, as causas cirúrgicas com média de $7,6 \%$, as causas genitourinárias, como infecção do trato urinário (ITU), insuficiência renal aguda (IRA) com média de 5,6\%, as causas cardiológicas, com média de $2,6 \%$, as causas neurológicas com média de 6,4\%, e as demais causas definidas, que, como o número é reduzido, foram agrupadas, tendo média de $11,8 \%$, num predomínio de hospitalizações para tratamento de doenças não crônicas. Apresenta-se a procedência segundo a causa da hospitalização na tabela 3 .

Tanto crianças oriundas do município de Cascavel, como aquelas de outros municípios que foram hospitalizadas no ACP do HUOP tiveram como causa preponderante as doenças respiratórias com 53\% e 37\%, respectivamente. Embora este

Tabela 2 - Causas de hospitalização em crianças menores de cinco anos, no ACP do HUOP, no período de 2005 a 2009 , Cascavel, PR, 2009.

\begin{tabular}{|c|c|c|c|c|c|c|c|c|c|c|c|c|}
\hline \multirow{2}{*}{$\begin{array}{l}\text { Período } \\
\text { Respiratória }\end{array}$} & \multicolumn{2}{|c|}{2005} & \multicolumn{2}{|c|}{2006} & \multicolumn{2}{|c|}{2007} & \multicolumn{2}{|c|}{2008} & \multicolumn{2}{|c|}{2009} & \multicolumn{2}{|c|}{ Total } \\
\hline & 50 & $53 \%$ & 102 & $56 \%$ & 101 & $57 \%$ & 54 & $61 \%$ & 49 & $53 \%$ & 356 & $56 \%$ \\
\hline Demais causas & 44 & $47 \%$ & 81 & $44 \%$ & 76 & $43 \%$ & 34 & $39 \%$ & 43 & $47 \%$ & 278 & $44 \%$ \\
\hline \multirow[t]{2}{*}{ Total } & 94 & $100 \%$ & 183 & $100 \%$ & 177 & $100 \%$ & 88 & $100 \%$ & 92 & $100 \%$ & 634 & $100 \%$ \\
\hline & \multicolumn{2}{|c|}{0,54} & \multicolumn{2}{|c|}{0,12} & \multicolumn{2}{|c|}{0,06} & \multicolumn{2}{|c|}{$0,03 *$} & \multicolumn{2}{|c|}{0,53} & \multicolumn{2}{|c|}{$0,00 *$} \\
\hline
\end{tabular}

*Significativo ao nível de $5 \%$ de probabilidade 
Tabela 3 - Procedência das crianças hospitalizadas no ACP do HUOP, de acordo com a causa da internação, no período de 2005 a 2009, Cascavel, PR, 2009.

\begin{tabular}{|c|c|c|c|c|}
\hline \multirow{2}{*}{ Causa da hospitalização } & \multicolumn{4}{|c|}{ Local de origem } \\
\hline & \multicolumn{2}{|c|}{ Cascavel (79\%) } & \multicolumn{2}{|c|}{ Outros (21\%) } \\
\hline Respiratória & 301 & $53 \%$ & 55 & $37 \%$ \\
\hline Desnutrição & 3 & $1 \%$ & 3 & $2 \%$ \\
\hline Desidratação & 5 & $1 \%$ & 1 & $1 \%$ \\
\hline Gastrointestinal & 39 & $7 \%$ & 5 & $3 \%$ \\
\hline Neonatal & 45 & $8 \%$ & 12 & $8 \%$ \\
\hline Causa externa & 8 & $1 \%$ & 5 & $3 \%$ \\
\hline Cirúrgica & 32 & $6 \%$ & 23 & $16 \%$ \\
\hline Hematológica & 10 & $2 \%$ & 0 & $0 \%$ \\
\hline Urológica & 24 & $4 \%$ & 5 & $3 \%$ \\
\hline Cardiológica & 8 & $1 \%$ & 4 & $3 \%$ \\
\hline Neurológica & 36 & $6 \%$ & 9 & $6 \%$ \\
\hline Outras & 63 & $10 \%$ & 26 & $17 \%$ \\
\hline Total & 574 & $100 \%$ & 148 & $100 \%$ \\
\hline
\end{tabular}

Tabela 4 - Tempo médio de internação em dias, segundo a faixa etária, no ACP do HUOP, por ano, no período de 2005 a 2009, Cascavel, PR, 2009.

\begin{tabular}{l|c|c|c|c|c|c}
\hline \multirow{2}{*}{ Faixa Etária } & \multicolumn{5}{|c|}{ Período } & $\begin{array}{c}\text { Média de } \\
\text { dias por } \\
\text { faixa etária }\end{array}$ \\
\cline { 2 - 6 } & $\mathbf{2 0 0 5}$ & $\mathbf{2 0 0 6}$ & $\mathbf{2 0 0 7}$ & $\mathbf{2 0 0 8}$ & $\mathbf{2 0 0 9}$ & 9,5 \\
\hline$<1$ ano & 8,9 & 11,4 & 9,6 & 9,0 & 8,5 & 9,5 \\
1 a $<5$ anos & 7,3 & 9,0 & 9,1 & 7,6 & 8,8 & 8,4 \\
Média no período & 8,1 & 10,2 & 9,3 & 8,3 & 8,6 & 8,9 \\
p-valor & $0,00 *$ & $0,00 *$ & $0,04 *$ & $0,00 *$ & 0,8 & $0,00 *$ \\
\hline
\end{tabular}

*Significativo ao nível de $5 \%$ de probabilidade

seja um hospital de referência regional, recebendo pacientes de uma área geográfica que compreende 25 municípios, nas hospitalizações infantis houve predomínio de internações de crianças do próprio município. Analisou-se ainda o tempo médio de duração das hospitalizações, apresentado na tabela 4.

Descreveu-se o tempo médio de internação de acordo com a divisão etária de menor de um ano, correspondendo a 9,5 dias e para crianças de um a menores de cinco anos, correspondendo a 8,4 dias. Fez-se a comparação do tempo de internação entre as duas faixas etárias por meio do Teste t-student de comparação de médias, sendo significativamente maior em todos os períodos, com exceção do ano de 2009, para a faixa etária de menor de um ano. Outro aspecto considerado foi o desfecho da hospitalização, cujos dados estão na tabela 5.

Quanto ao desfecho da hospitalização, incluindo altas, transferências e óbitos, obteve-se 95\% para alta, 3,0\% de transferências para outras instituições ou mesmo outras unidades do hospital (UTI pediátrica e/ou neonatal) e 2,0\% de óbitos, correlacionado a causa de hospitalização.

Tabela 5 - Desfecho da hospitalização de acordo com a causa da internação, no ACP do HUOP, no período de 2005 a 2009, Cascavel, PR, 2009.

\begin{tabular}{|c|c|c|c|c|c|c|}
\hline \multirow{3}{*}{$\begin{array}{l}\text { Causa } \\
\text { Respiratória }\end{array}$} & \multicolumn{6}{|c|}{ Desfecho da hospitalização } \\
\hline & \multicolumn{2}{|c|}{ Alta $(95 \%)$} & \multicolumn{2}{|c|}{ Óbito (2,0\%) } & \multicolumn{2}{|c|}{ Transferido $(3,0 \%)$} \\
\hline & 348 & $51 \%$ & 3 & $18 \%$ & 4 & $22 \%$ \\
\hline Desnutrição & 6 & $1 \%$ & 0 & $0 \%$ & 0 & $0 \%$ \\
\hline Desidratação & 6 & $1 \%$ & 0 & $0 \%$ & 0 & $0 \%$ \\
\hline Gastrointestinal & 44 & $6 \%$ & 0 & $0 \%$ & 0 & $0 \%$ \\
\hline Neonatal & 52 & $8 \%$ & 1 & $6 \%$ & 4 & $22 \%$ \\
\hline Causa externa & 11 & $2 \%$ & 0 & $0 \%$ & 2 & $11 \%$ \\
\hline Cirúrgica & 54 & $8 \%$ & 0 & $0 \%$ & 0 & $0 \%$ \\
\hline Hematológica & 10 & $1 \%$ & 0 & $0 \%$ & 0 & $0 \%$ \\
\hline Urológica & 27 & $4 \%$ & 0 & $0 \%$ & 2 & $11 \%$ \\
\hline Cardiológica & 7 & $1 \%$ & 3 & $18 \%$ & 2 & $11 \%$ \\
\hline Neurológica & 41 & $6 \%$ & 3 & $18 \%$ & 1 & $6 \%$ \\
\hline Outras & 78 & $11 \%$ & 7 & $41 \%$ & 3 & $17 \%$ \\
\hline Total & 684 & $100 \%$ & 17 & $100 \%$ & 18 & $100 \%$ \\
\hline
\end{tabular}

\section{DISCUSSÃO}

O presente estudo utilizou exclusivamente dados de registro dos prontuários hospitalares. Este tipo de dado é uma importante ferramenta para análises estatísticas dos casos que levam a internação, entretanto, o uso destas estatísticas tem limitações, pois são dados secundários, e algumas precauções devem ser tomadas na realização de inferências sob o ponto de vista epidemiológico.

Ao se utilizar as estatísticas hospitalares, a principal limitação encontrada é a seletividade, já que o sistema não é universal e as internações em hospitais privados não são incluídas. Logo, os resultados refletem tendências e padrões das morbidades somente para a população assistida pelo SUS. Em um hospital universitário ocorre também a limitação do 
registro, relacionado tanto a variedade de profissionais quanto a diversidade de acadêmicos dos diversos cursos de saúde que manuseiam e realizam registros nos prontuários, nem sempre de forma adequada.

Os dados desse estudo indicam que o diagnóstico majoritário para hospitalização foi aquele do grupo de doenças respiratórias, a exemplo de diversos estudos que vem sendo realizados em nossa realidade há pelo menos duas décadas. Assim, pode-se definir o perfil encontrado para hospitalizações nessa pesquisa como: predominância de internações de menores de um ano (42\%), do sexo masculino (49,6\%), por causas respiratórias $(49,6 \%)$, procedentes do próprio município (53\%), com tempo médio de hospitalização de 9,5 dias e com $95 \%$ de desfecho para alta hospitalar.

Estudo $^{(5)}$ realizado em Sobral, no Ceará, para descrever a morbidade referida em crianças de 5 a 9 anos e analisar seus possíveis condicionantes aponta que, das crianças participantes do estudo, 42,9\% apresentaram morbidade referida, sendo a causa mais frequente dos problemas de saúde relatados, classificados pela CID-10, as doenças do aparelho respiratório $(28,7 \%)$, com prevalência cinco vezes maior que o segundo grupo de morbidade referida mais prevalente, os sinais e sintomas não classificados em outra parte.

Estudos $^{(6-7)}$ apontam que, enquanto nos países desenvolvidos as IRA têm importante participação na morbidade em crianças menores de cinco anos, nos países em desenvolvimento essas infecções tendem a ser mais graves e operam com componente considerável tanto na morbidade quanto na mortalidade, concentrando cerca de 20 a $40 \%$ das consultas em serviços de pediatria, 12 a 35\% das internações hospitalares e $19 \%$ das mortes.

Em estudo ${ }^{(8)}$ para caracterizar a morbidade hospitalar no município de Maringá - PR, em 1992, na população em geral, entre os diagnósticos mais importantes encontrou-se doenças do aparelho respiratório. Das internações, 42,8\% foram de pacientes do sexo masculino e, quando ajustadas por idade, concentrou-se em crianças de até quatro anos, sendo os diagnósticos mais comuns nessa faixa de idade também o grupo das doenças do aparelho respiratório. Esses achados corroboram os de nosso estudo, com predomínio para o sexo masculino e também da faixa etária de menor de um ano como mais susceptível.

Em estudo ${ }^{(9)}$ realizado em Cuiabá, sobre a morbidade por pneumonia em crianças menores de cinco anos, os resultados mostraram como marcante a frequência da IRA em menores de cinco anos, pois quase a metade $(49,8 \%)$ dos atendimentos desta faixa etária ocorreu por sinais e sintomas decorrentes de algum diagnóstico de IRA, ficando todos os outros diagnósticos (diarréia, dermatoses, traumas, intoxicações exógenas, etc.) com a outra metade, assim como ocorreu em nossa pesquisa, cuja causa predominante de hospitalização foi por doenças do grupo respiratório, preponderantemente as pneumonias.

No município de Pelotas, RS, estudo(10) realizado para avaliar fatores de risco para hospitalização por doença respiratória aguda em crianças até um ano de idade, trouxe como resultados, em relação às características demográficas, predomínio do sexo masculino na amostra em geral (55,6\%). A média de idade e desvio-padrão dos casos e dos controles foram, respectivamente, de 5,3 $\pm 3,0$ meses e de 6,2 $\pm 3,2$ meses e $64,9 \%$ do total da amostra apresentava menos de seis meses de idade. As causas mais frequentes de hospitalizações foram: pneumonia $(43,7 \%)$, bronquiolite $(31,0 \%)$, asma $(20,3 \%)$, gripe $(3,5 \%)$, otite média aguda $(0,8 \%)$ e laringite $(0,6 \%)$.

Acompanhou-se cerca de 11.000 crianças pertencentes a duas coortes de base populacional na cidade de Pelotas - RS, em 1982 e 1993, para medir a ocorrência de hospitalizações e sua associação com peso ao nascer e renda familiar. Cerca de $20 \%$ das crianças foram hospitalizadas pelo menos uma vez no primeiro ano de vida e os meninos foram os mais acometidos, entre outros achados ${ }^{(11)}$.

Em 1994, um estudo(12) realizado no Maranhão, revelou que a taxa de hospitalização infantil foi de $24,4 \%$, sendo as maiores por pneumonia $(7,3 \%)$ e diarréia $(7,1 \%)$. Outro estudo $^{(13)}$ realizado na região metropolitana de São Paulo, em 1996, mostrou que das crianças que foram hospitalizadas, 41,5\% apresentavam doenças do aparelho respiratório, com ênfase para infecção respiratória aguda $(27,7 \%)$. Os dados encontrados em nosso estudo, uma década depois, corroboram os apresentados pela literatura citada, indicando que as medidas adotadas até o momento na atenção a saúde das crianças não tem mudado esse quadro.

Estudo $^{(14)}$ com crianças de um mês a 14 anos, com diagnóstico de pneumonia adquirida na comunidade, internadas no Hospital Nossa Senhora da Conceição, em Porto Alegre, aponta como resultado uma média de idade de 2,3 anos, predominando o gênero masculino em $59 \%$. Constatou que poucas crianças possuíam vacinação contra o pneumococo, refletindo a necessidade de inclusão das vacinas antipneumocócicas no calendário vacinal.

Analisou-se as internações hospitalares por doenças respiratórias em menores de 15 anos de idade em Tangará da Serra (MT), no período de 2000 a $2005^{(15)}$. Revelou-se que a taxa de internações por doenças respiratórias em menores de 15 anos foi de 70,1/1.000 crianças, sendo 12.777 internações de crianças, das quais $8.142(63,7 \%)$ por doenças respiratórias. As principais causas de internação foram: pneumonias $(90,7 \%)$ e insuficiência respiratória $(8,5 \%)$. Em menores de 5 anos de idade, as internações por pneumonia foram mais de 4 vezes o esperado para o município. Os menores de 12 meses de idade tiveram mais internações, em média 32,4 internações por 1.000 crianças a cada ano.

Em estudo realizado para estabelecer a etiologia das infecções respiratórias agudas e epidemiologia dos vírus respiratórios mais comuns em crianças pequenas atendidas no hospital universitário da Universidade de São Paulo, em 2003, observou-se que as crianças menores de cinco anos, especialmente as menores de um ano, apresentaram uma alta taxa de hospitalização devido aos vírus respiratórios sincicial humano, influenza, parainfluenza 1, 2 e 3, metapneumovírus humano e adonovírus, recomendando estratégias de prevenção a partir desse conhecimento ${ }^{(16)}$.

No município de Ribeirão Preto, SP, estudo(17) revelou que os dados sobre morbidade hospitalar indicavam entre os grupos de diagnósticos mais frequentes nas internações de 
menores de um ano, as doenças do aparelho respiratório, recomendando que a intervenção tecnicamente adequada sobre estas patologias implicaria, basicamente, no desenvolvimento de ações em outros níveis que não o hospitalar, entendida como referência aos serviços de atenção primária em saúde.

Quase trinta anos após esse estudo e muitas mudanças no sistema de saúde, assim como estratégias específicas para o grupo infantil, os dados permanecem praticamente inalterados, com predomínio das doenças respiratórias nas causas de hospitalização, indicando que as ações voltadas a esse grupo não têm conseguido mudar o panorama das hospitalizações infantis.

Com relação ao indicador de mortalidade, em estudo ${ }^{(18)}$ de revisão das infecções respiratórias por vírus sincicial respiratório em crianças, a taxa de mortalidade foi de 3,8\% nos pacientes hospitalizados com pneumonia ou bronquiolite, menor do que aquela encontrada em nosso estudo, de $18 \%$ para as doenças respiratórias, mas maior do que aquela apreendida para todas as patologias, que foi de $2 \%$, no conjunto das hospitalizações.

Em estudo ${ }^{(19)}$ realizado para conhecer o perfil epidemiológico das internações pediátricas em unidade de terapia intensiva pediátrica em Maringá, no período de janeiro de 2004 a janeiro de 2006, encontrou-se que a maior parte das hospitalizações era de pacientes do sexo masculino (55,74\%), lactentes $(50,71 \%)$ e procedentes de outros municípios $(64,90 \%)$. O tempo médio de internação para $82,21 \%$ dos casos foi menos de dez dias e as causas mais frequentes de internação foram as afecções do aparelho respiratório $(41,38 \%)$ e as lesões, envenenamentos e causas externas $(9,60 \%)$. A taxa de mortalidade foi de 6,94\%.

Esses achados corroboram os de nosso estudo, em que a maior parte dos pacientes foi do sexo masculino, as causas mais frequentes de hospitalização foram as doenças respiratórias, a média de dias de hospitalização foi inferior a dez dias. Difere o percentual de óbitos, menor em nosso estudo e a procedência dos pacientes. Embora as duas instituições tenham abrangência regional, houve predomínio, em nosso estudo de hospitalização do município sede da instituição, o que pode ter sido influenciado pelo fato de serem unidades de internação com perfis diferentes.

O perfil de morbidade infantil permite conhecer a realidade dos fatores que determinam a ocorrência e distribuição de saúde e doença nessa população. Assim, ao comparar os dados obtidos localmente pode-se perceber que os mesmos são correspondentes aos dados de outras localidades no país trazidos por diversos autores e também aos nacionais, discutidos pelas autoras em artigo publicado anteriormente ${ }^{(20)}$. Tal fato reforça a importância de olhar especialmente as ações de prevenção e promoção da saúde infantil que evitem os agravos respiratórios ou possibilitem o tratamento adequado no nível da atenção básica, uma vez que esse grupo de doenças faz parte das causas sensíveis à atenção ambulatorial, sem necessidade de hospitalização para seu adequado manejo.

Trazemos a seguir estudos, tanto no âmbito do hospital quanto da atenção básica, que reforçam a importância de utilizarem-se dados epidemiológicos no planejamento de ações de saúde.

Em estudo ${ }^{(21)}$ cujo objetivo foi apresentar o panorama das internações sensíveis à atenção primária no Estado de São
Paulo, no período de 2000 a 2007, observou-se redução nas internações por diabetes e chamou atenção o aumento de internações por doenças relacionadas ao pré-natal e parto. Referem que estudos que se utilizem de abordagem que resulte na produção de dados podem ser de grande valia para os gestores envolvidos com a atenção primária, podendo funcionar como balizadores de sua qualidade contribuindo para avaliação da implantação das políticas de saúde.

Estudo $^{(22)}$ realizado no âmbito do hospital com profissionais enfermeiros, a fim de avaliar a qualidade do cuidado, utilizou-se de indicadores de qualidade do serviço para instrumentalizar as ações de enfermagem em busca de melhoria do cuidado. A respeito do uso de dados epidemiológicos e de avaliação do serviço para embasamento da prática, verificou-se que $68 \%$ da amostra estudada concordou que os resultados dos indicadores utilizados no setor efetivamente demonstram melhoria da prática assistencial. Quanto a utilização dos mesmos resultados dos indicadores para embasamento da gestão do setor, 79\% da amostra estudada concordou com a afirmativa. Assim, os resultados demonstraram que os indicadores implantados nos setores assistenciais não só contribuíram para a melhoria da prática assistencial, mas também para tomadas de decisão setoriais e foram instrumentos essenciais para a implementação de ações de melhoria.

Outro estudo ${ }^{(23)}$ identificou nas publicações de periódicos nacionais e internacionais os principais padrões de dados, terminologias e sistemas de classificação utilizados no cuidado em saúde e Enfermagem e, a partir dos diversos padrões de dados, terminologias e sistemas existentes, considerou importante que a Enfermagem se aproprie dos mesmos visando aprimorar e renovar a qualidade do cuidado, pois a informação é essencial para o cuidado de Enfermagem, subsidiando o enfermeiro na tomada de decisão clinica para a resolução e diminuição dos problemas em saúde.

Com o objetivo de avaliar a efetividade da aplicação de evidências pela pesquisa-ação-participante (PAR) em uma unidade de enfermagem observou-se melhora significativa na qualidade dos registros de enfermagem na avaliação de sinais e sintomas do paciente, o ajuste psicossocial do paciente melhorou significativamente, concluindo-se que a PAR tem servido para apresentar evidências e melhorar os resultados de saúde ${ }^{(24)}$.

Além do uso de indicadores na prática hospitalar, na rede de atenção básica são largamente produzidos indicadores sobre a assistência prestada. Utilizar esses indicadores, a exemplo do estudo(25) realizado para verificar a cobertura de vacinação infantil e sua relação com a mortalidade, no município de Campina Grande, mostra a efetividade dessa prática por enfermeiros, que podem mudar sua realidade a partir do uso desses dados no planejamento e desenvolvimento de ações. Os autores apontaram que a identificação da cobertura vacinal e dos fatores responsáveis pelo retardo ou pela falta de imunizações foi ação fundamental para a adequada monitorização dos programas de vacinação e para se identificar e atingir as crianças que não são vacinadas.

Em relação ao ambiente hospitalar, conhecer os dados de perfil permite planejar o cuidado de enfermagem com propriedade, desde a quantidade de recursos humanos necessários 
até a qualificação de pessoal com foco na qualidade do cuidado, na diminuição do tempo de permanência da criança no hospital e na implementação da sistematização da assistência de enfermagem. Os dados mostram que mais de $50 \%$ das hospitalizações são de doenças do grupo respiratório, assim, é possível investir na capacitação da equipe de enfermagem para o cuidado a essas doenças, identificando precocemente sinais de piora, efetivando medidas de conforto para os sintomas e, por meio da qualidade do cuidado, reduzindo as consequências da hospitalização.

No âmbito da atenção básica, recomenda-se que os programas voltados à saúde da criança sejam assumidos pelo enfermeiro como prioritários, iniciando-se com a atenção pré e perinatal. Embora não tenhamos identificado os fatores prematuridade ou baixo peso no estudo como determinantes das hospitalizações, enfocar essas duas características é importante, pois são sabidos fatores de risco para o adoecimento. A assistência à criança com IRA na unidade ambulatorial deve incluir a educação em saúde da população para reconhecimento dos sinais de gravidade, bem como a capacitação de recursos humanos no manejo adequado desse grupo de doenças, pois a amplitude na cobertura e na qualidade da atenção ambulatorial tem o potencial de reduzir as taxas de hospitalização e consequentemente os efeitos prejudiciais que esta tem sobre a criança e sua família.

Com ações como a realização da puericultura até os dois anos de idade, acompanhamento do crescimento e desenvolvimento até os cinco anos, avaliação das crianças nas escolas, visitas domiciliares, incentivo a amamentação, cobertura ampla de imunizações, que são as práticas cotidianas das unidades de saúde, sejam saúde da família ou unidades básicas tradicionais, é possível intervir nesse indicador. A detecção precoce dos sinais e sintomas respiratórios, a exemplo do preconizado na estratégia AIDPI, pois estes podem se agravar rapidamente em crianças evoluindo para as pneumonias, é outro aspecto a ser considerado. Ter uma unidade de referência na rede de serviços, para o encaminhamento ágil e resolutivo também é um fator que minimiza o agravamento do quadro respiratório.

\section{CONCLUSÃO}

Embora possa parecer óbvio, a atenção dispensada pelos serviços de saúde destinados à atenção primária poderia ser mais efetiva, a partir da intervenção sobre os fatores de risco sabidamente relacionados com o processo de acometimento das doenças respiratórias. Sabendo-se que as causas majoritárias de hospitalização estão no agrupamento de doenças respiratórias, o enfermeiro que atua na atenção básica precisa direcionar suas ações preventivas as crianças menores de cinco anos no sentido de minimizar esse problema. Quando a hospitalização ocorre, a enfermagem deve atuar no sentido de auxiliar na recuperação da criança o mais breve possível e prepará-la para o retorno a atenção básica para a continuidade dos cuidados após o período de hospitalização almejando a redução das recidivas, constituindo-se em importante determinante para garantir um crescimento saudável das crianças abaixo de cinco anos. Essa medida pode se tornar efetiva com a contrarreferência do enfermeiro do hospital para aquele da unidade de atenção básica, colocando para funcionar uma rede de cuidados a criança que previna novos adoecimentos.

Parece-nos oportuno, portanto, a execução de mais estudos objetivando reconhecer e avaliar a magnitude dos fatores de suscetibilidade da criança ao adoecimento, contribuindo para práticas em saúde que considerem os dados epidemiológicos em seu planejamento de ações e possam contribuir com a efetividade do cuidado em saúde.

\section{REFERÊNCIAS}

1. Malucelli A, Otemaier KR, Bonnet M, Cubas MR, Garcia, TR. Sistema de informação para apoio à Sistematização da Assistência de Enfermagem. Rev Bras Enferm 2010;63(4):629-36.

2. Ministério da Saúde (Brasil). Portaria GM n 2395, de 7 de outubro de 2009. Estratégia Brasileirinhas e Brasileirinhos Saudáveis. Diário Oficial da União 9 out 2009. Seção 1.

3. Cardoso AM. A persistência das infecções respiratórias agudas como problema de saúde pública. Cad Saúde Pública 2010;26(7):1270.

4. Rede Interagencial de Informação para a saúde (RIPSA). Indicadores básicos para a saúde no Brasil: conceitos e aplicações. Brasília: OPAS; 2008.

5. Barreto ICHC, Grisi SJFE. Morbidade referida e seus condicionantes em crianças de 5 a 9 anos em Sobral, CE, Brasil. Rev Bras Epidemiol 2010;13(1):35-48.

6. Pinto KDBPC, Maggi RRS, Alves JGB. Análise de risco socio-ambiental para comprometimento pleural na pneumonia grave em crianças menores de 5 anos. Rev Panam Salud Publica 2004;15(2):104-9.

7. UNICEF [homepage na internet]. Situação mundial da infância 2008. [acesso em 23 ago 2010]. Disponível em: < http:// www.unicef.org/brazil/sowc2008final/index.html>

8. Mathias IAI, Soboll MIM. Morbidade hospitalar em município da região Sul do Brasil em 1992. Rev Saúde Pública 1996;30(3):224-32.

9. Botelho C, Correia AL, Silva AMC, Macedo AG, Silva COS. Fatores ambientais e hospitalizações em crianças menores de cinco anos com infecção respiratória aguda. Cad Saúde Pública 2003;19(6):1771-80.

10. Macedo SEC, Menezes AMB, Albernaz E, Post, P, Knorst $M$. Fatores de risco para internação por doença respiratória aguda em crianças até um ano de idade. Rev Saúde Pública 2007;41(3):351-8.

11. César JA, Victora CG, Barros FC, Ramos FA, Albernaz EP, Oliveira LM, et al. Hospitalizações em menores de um ano pertencentes a duas coortes de base populacional no 
Sul do Brasil: tendências e diferenciais. Cad Saúde Pública 1996;(12 Supl 1):67-71.

12. Silva AAM, Gomes UA, Tonial SR, Silva RA. Fatores de risco para hospitalização de crianças de um a quatro anos em São Luís, Maranhão, Brasil. Cad Saúde Pública 1999;15(4):749-57.

13. Caetano JRM. Bordin IAS, Puccini RF, Peres CA. Fatores associados à internação hospitalar de crianças menores de cinco anos, São Paulo, SP. Rev Saúde Pública 2002;36(3):285-91.

14. Jock CLM, Sakae TM, Dal-Bó K. Pneumonias na enfermaria de pediatria do Hospital Nossa Senhora Conceição, em Tubarão - SC. Arq Catarinenses Med 2009;38(1):7-13.

15. Rosa AM, Ignotti E, Hacon SS, Castro HÁ. Análise das internações por doenças respiratórias em Tangará da Serra Amazônia Brasileira. J Bras Pneumol 2008;34(8):575-82.

16. Thomazelli LM, Vieira S, Leal AL, Sousa TS, Oliveira DBL, Golono MA, et al. Vigilância de oito vírus respiratórios em amostras clínicas de pacientes pediátricos no sudeste do Brasil. J Pediatr 2007;83(5):422-8.

17. Barros MBA. Morbidade e mortalidade hospitalar de crianças menores de um ano, em Ribeirão Preto, SP (Brasil), 1975. Rev Saúde Pública 1981;15(3):308-20.

18. Lourenção LG, Salomão Júnior JB, Rahal P, Souza FP, Zanetta DMT. Infecções pelo Vírus Sincicial Respiratório em crianças. Pulmão 2005;14(1):59-68.
19. Molina RCM, Marcon SS, Uchimura TT, Lopes EP. Caracterização das internações em uma Unidade de Terapia Intensiva Pediátrica, de um hospital-escola da região sul do Brasil. Ciênc Cuid Saude 2008;(7 Supl 1):112-20.

20. Oliveira BRG, Viera CS, Collet N, Lima RAG. Causas de hospitalização no SUS de crianças de zero a quatro anos no Brasil. Rev Bras Epidemiol 2010;13(2):268-77.

21. Rehem TCMSB, Egry EY. Internações por Condições Sensíveis à Atenção Primária no Estado de São Paulo. Ciênc Saúde Coletiva 2011;16(12):4755-66.

22. Franco JN, Barros BPA, Vaidotas M, D'innocenzo M. Percepção dos enfermeiros sobre os resultados dos indicadores de qualidade na melhoria da prática assistencial. Rev Bras Enferm 2010;63(5):806-10.

23. Barra DCC, Sasso GTMD . Padrões de dados, terminologias e sistemas de classificação para o cuidado em saúde e enfermagem. Rev Bras Enferm 2011;64(6):1141-9.

24. Abad-Corpa E, Cabrero-García J, Delgado-Hito P, Carrillo-Alcaraz A, Meseguer-Liza C, Martínez-Corbalán JT. Avaliação da efetividade da aplicação de evidências por meio de pesquisa-ação-participante em uma unidade de enfermagem hematológica. Rev Latino-Am Enferm 2012;20(1).

25. Franca ISX, Simplicio DN, Alves FP, Brito VRS. Cobertura vacinal e mortalidade infantil em Campina Grande, PB, Brasil. Rev Bras Enferm 2009;62(2):258-71. 